\title{
A Pendant-drop-Type Tensiometer for Surface Tension Measurements of Polymer Systems
}

\author{
Akio Nakajima and Masahiko Murakami \\ Department of Polymer Chemistry, Kyoto University \\ Sakyo-ku, Kyoto, Japan.
}

(Received January 6, 1972)

\section{KEY WORDS Surface Tension / Pendant-drop-Type Tensiometer /}

Due to the experimental difficulties in performing measurements on viscous liquids, studies on surface and interfacial tensions of polymer systems at the solution-air, melt-air, and meltmelt interfaces have not been widely extended. Among the various methods to measure surface tension, techniques such as the capillary rise, the du Noüy ring, the drop-weight or dropvolume, and the maximum bubble pressure methods may not be suited for polymers on account of the high viscosity of such systems. To the contrary, the pendant-drop method and the sessible-bubble method seem to be prominent for surface tension measurements of polymeric systems. Thus, Frisch, ${ }^{1}$ Roe, ${ }^{2,3}$ and $\mathrm{Wu}^{4}$ have promoted the former method, originally proposed by Andreas, ${ }^{5}$ and $\mathrm{Hata}^{6}$ refined the latter method, which was first applied to polymer melt by Sakai. $^{7}$ In this note we report on a pendant- drop type tensiometer designed in our laboratory.

Figure 1 illustrates the whole assembly of the apparatus. A light beam from a $\mathrm{Hg}-2$ type mercury arc lamp (A) was filtered to isolate 546-m $\mu$ light and parallelized through a filter (B), and entered in the pendant-drop cell (C). (D) is an objective lens of $f=4.5$ and $105-\mathrm{mm}$ focal length, (E) is a bellows, (F) is a $35-\mathrm{mm}$ Camera, and $(\mathbf{H})$ is the optical bench. In the camera $(F)$, a fine thread $(G)$ is suspended in front of the film plane to detect the vertical direction. Details of the pendant-drop cell is shown in Figure 2, in which (I) is micrometer, (J) spring, (K) quartz plunger, (L) quartz syringe, (M) quartz cell, (N) sample, (O) pendant-drop of the sample, (P) quartz windows, (Q) aluminum heating block, (R) supporting sphere, (S) screws, (T) holder, and (U) temperature adjustment.

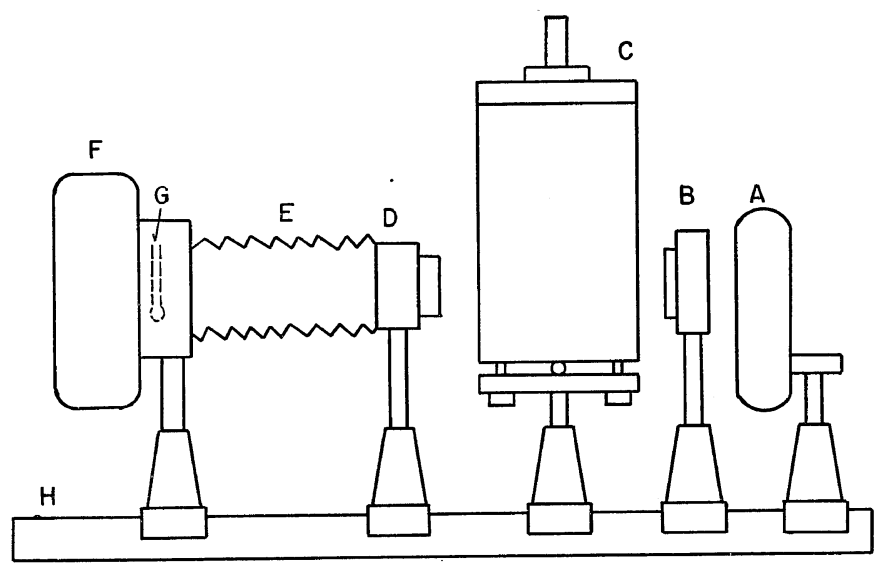

Figure 1. Apparatus. 


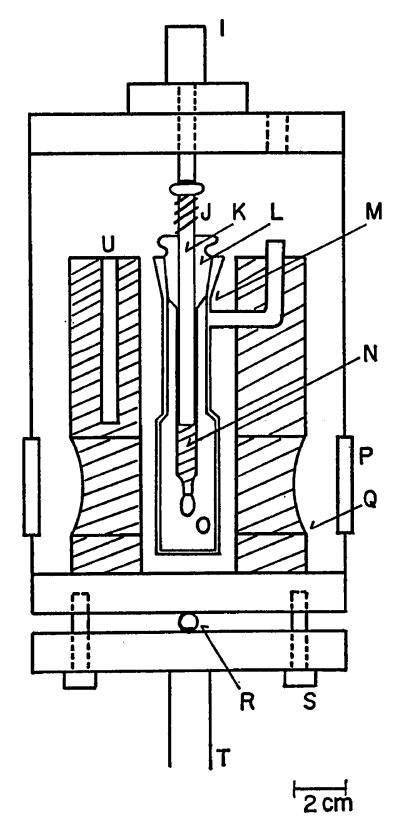

Figure 2. Pendant-drop cell.
The pendant-drop of the sample was formed by sliding the plunger by the micrometer with the aid of the spring. The screws (S) serve to adjust the position of pendant-drop to facilitate focusing and alignment of the pendant-drop.

Prior to measurement with a sample, a stainless steel tube with known diameter was inserted in the cell in place of the syringe. The end of the tube was fixed at the same position as

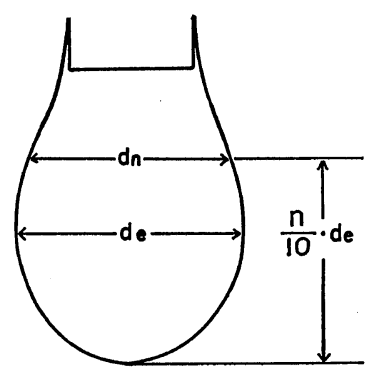

Figure 3. Dimension of a pendant-drop.

Table I. Surface tension values of toluene, $n$-heptane, and poly(dimethylsiloxane)

\section{(a) Toluene $\left(24.5^{\circ} \mathrm{C}\right)$}

$\left(\Delta \rho, 0.8616 \mathrm{~g} / \mathrm{cm}^{3} ; d_{e}, 0.2421 \mathrm{~cm}\right)$

\begin{tabular}{lccccc}
\hline \multicolumn{1}{c}{$n$} & 8 & 9 & 10 & 11 & 12 \\
\hline$S_{n}$ & 0.9336 & 0.8738 & 0.7994 & 0.7202 & 0.6444 \\
$1 / H$ & 0.5765 & 0.5675 & 0.5665 & 0.5654 & 0.5665 \\
$\gamma$, dyn/cm & $(28.5)$ & 28.1 & 28.1 & 28.0 & 28.1 \\
$\gamma($ av. $), 28.1 \mathrm{dyn} / \mathrm{cm}$ & & & & & \\
$\gamma\left(\right.$ lit. $\left.^{12}\right), 28.0 \mathrm{dyn} / \mathrm{cm}$ & & & & & \\
\hline
\end{tabular}

(b) $n$-Heptane $\left(24.6^{\circ} \mathrm{C}\right)$

$\left(\Delta \rho, 0.6787 \mathrm{~g} / \mathrm{cm}^{3} ; d_{e}, 0.2336 \mathrm{~cm}\right)$

\begin{tabular}{lccccc}
\hline \multicolumn{1}{c}{$n$} & 8 & 9 & 10 & 11 & 12 \\
\hline$S_{n}$ & 0.9382 & 0.8793 & 0.8078 & 0.7319 & 0.6613 \\
$1 / H$ & 0.5513 & 0.5510 & 0.5513 & 0.5509 & 0.5509 \\
$\gamma$, dyn/cm & 20.0 & 20.0 & 20.0 & 20.0 & 20.0 \\
$\gamma($ av. $), 20.0 \mathrm{dyn} / \mathrm{cm}$ & & & & \\
$\gamma\left(\right.$ lit. $\left.^{2}\right), 19.8 \mathrm{dyn} / \mathrm{cm}$ & & & & \\
\hline
\end{tabular}

(c) Poly(dimethylsiloxane) $\left(23.8^{\circ} \mathrm{C}\right)$

$\left(\Delta \rho, 0.9688 \mathrm{~g} / \mathrm{cm}^{3} ; d_{e}, 0.2137 \mathrm{~cm}\right)$

\begin{tabular}{lccccc}
\multicolumn{1}{c}{$n$} & 8 & 9 & 10 & 11 & 12 \\
\hline$S_{n}$ & 0.9563 & 0.9133 & 0.8593 & 0.8091 & 0.7630 \\
$1 / H$ & 0.4659 & 0.4633 & 0.4679 & 0.4658 & 0.4682 \\
$\gamma$, dyn/cm & 20.2 & 20.1 & 20.3 & 20.2 & 20.3 \\
$\gamma\left(\right.$ av. $^{2}, 20.2$ dyn/cm & & & & \\
$\gamma\left(\right.$ lit. $\left.^{2}\right), 20.2$ dyn/cm (extrapolated to $23.8^{\circ} \mathrm{C}$ ) & & & & \\
\hline
\end{tabular}




\section{A. Nakajima and M. Murakami}

that expected for pendant-drop, and photographed on a film in the camera. Magnification of the image of pendant-drop was determined from the ratio of the diameter of photographic image of the tube to the diameter of the actual tube. After drawing out the stainless steel tube, the sample was introduced in the syringe, and image of the pendant-drop was photographed on film in the camera.

The surface tension $\gamma$ is estimated from the following equation ${ }^{5}$

$$
\gamma=\frac{g \Delta \rho\left(d_{e}\right)^{2}}{H}
$$

where, $g$ is the gravitational acceleration, $\Delta \rho$ the density difference between the liquid of drop and the surrounding medium, $d_{e}$ the largest horizontal diameter of the drop, and $H$ is a function of $S_{n}\left(=d_{n} / d_{e}\right)$, in which $d_{n}$ is the horizontal diameter at a distance equal to $d_{e}(n / 10)$ from the bottom of the drop (see Figure 3 ). Fordham, ${ }^{8}$ Niederhauser and Bartell, ${ }^{9}$ and Stauffer ${ }^{10}$ determined $1 / H$ as a function of $S_{n}$ for $n=10$, and Roe, et al. ${ }^{3}$ calculated $1 / H$ for $n=8,9,10,11$, and 12 by means of RungeKutta's method. Details of the results of numerical calculation were published by Roe. ${ }^{11}$

In this work, we measured $d_{e}$ and $d_{n}$ 's for $n=8,9,10,11$, and 12 , and determined the surface tension $\gamma$ by eq 1 from the values of $1 / H$ obtained using Roe's tables listing $1 / H$ as a function of $S_{n}$. Measurements of $d_{e}$ and $d_{n}$ 's were performed by the use of a Nikon Profile Projector Model V-16.

In Table I are summarized the surface tension values calculated from $d_{n}$ values at different $n(=8,9,10,11$, and 12$)$ for toluene, $n$-heptane, and poly(dimethylsiloxane) (Shinetsu Silicone
KF96).

As obvious from the table, numerical values of $\gamma$ calculated from different $d_{n}$ values are in accord each other and, further, the average value of $\gamma$ is in excellent agreement with literature values for the corresponding compounds. Thus, we conclude that the apparatus reported here is useful for measurements of polymeric systems with high accuracy.

Acknowledgement. The authors wish to thank Mr. Hiroshi Shirahase of Shimadzu Seisakusho Ltd., Kyoto, for his cooperation in manufacturing the apparatus.

\section{REFERENCES}

1. H. L. Frisch and S. Al-Madfai, J. Amer. Chem. Soc., 80, 3561 (1958).

2. R. J. Roe, J. Phys. Chem., 72, 2013 (1968).

3. R. I. Roe, V. L. Bacchetta, and P. H. G. Wong, J. Phys. Chem., 71, 4190 (1967).

4. S. Wu, J Colloid Interfac. Sci., 31, 153 (1969).

5. J. M. Andreas, E. A. Hauser, and W. B. Tucker, J. Phys. Chem., 42, 1001 (1938).

6. T. Hata, Surface (in Japanese), 6, 281 (1968).

7. T. Sakai, Polymer, 6, 659 (1965).

8. S. Fordham, Proc. Roy. Soc., A194, 1 (1948).

9. D. O. Niederhauser and F. E. Bartell, "Report of Progress Fundamental Research on Occurrence and Recovery of Petroleum 1948-1949," American Petroleum Institute, Baltimore, Md., 1950, p 114-146.

10. C. E. Stauffer, J. Phys. Chem., 69, 1933 (1965).

11. R. J. Roe, Document No. 9668, ADI Auxiliary Publications Project, Photoduplication Service, Library of Congress, Washington 24, D.C. 20540.

12. Organic Solvents, Physical Properties and Methods of Purification (1955). 